Gastroenterologia. 1953;79:I-IV

\title{
Contents, Vol. 79, 1953
}

\section{INDEX}

Ardran, G. M., F. H. Kemp and S. C. Truelove, A Radiological Study of the

Movements of the Tongue, Pharynx and Oesophagus in Acrosclerosis 361

Ardran, G. M., J. Badenoch, F. H. Kemp and W. C. D. Richards, Deformity

of the Lower End of the Oesophagus and the Adjacent Part of the Stomach

in Pernicious Anaemia. A Preliminary Communication 376

Badenoch, J., and W. C. D. Richards, The Gastric Lesion in Anaemia with Par

ticular Reference to Biopsy 329

Badenoch, J., vide G. M. Ardran

Bauer, Hans A., Hepatopathie und Magendarmkrankheiten

Bíkích, G., vide I. Faredin

Blake, J., Haeinatological and Nutritional Effects of Operation on the Stomach 337

Blöchlinger, L., vide S. Laser

Borbola, J., vide I. Faredin

Brummer, Pekka, and Penttí Koskínen, The Hyperventilation Test in Gastro-

duodenal and Neurocirculatory Dystonia 294

Chandler, G. N., and G. Watkinson, The Diagnostic Value of Gastric Aspiration

in Haematemesis 355

Cloesges, Joseph und Leo Norpoth, Hepatolenticuläres Syndrom im Alter .... 299

Cox, E. V., vide C. C. Ungley

Cuthbertson, W. F. J., Haeinatological and Nutritional Effects of Oral Anti

biotics 327

Davidson, L. S. P., Megaloblastic Anaemia and Subacute Combined Degenera

tion from Tuberculous Disease of the Small Intestine 342

Decker, P., et P. Francioli: Le traitement chirurgical du carcinome de Гæso-

phage et du cardia 117

Deucher, F., Einzeitige Resektion einer mehrfachen, innern und äußern Dünn-

Darm-Colonfistel 106

Dickinson, P. H., Circulation of Blood in the Rat's Stomach

Faredin, I., G. Bikich and J. Borbola, Determination of Histamin in Human

Gastric Juice 185

Francioli, P., vide P. Decker

Frercks, R, Beitrag zur Klinik der neurogenen Magentumoren 214

Fríedrich, L., Beiträge zur Frage des Magenschleimhautprolapscs 27

Hirschberg, Fritz, The Dumping Syndrome, Hypoglycemia and Jejunitis 16

Ihre, Bengt J. E., Routine Sigmoidoscopy for the Early Detection of Rectal

Carcinoma 347

Jentzer, Albert, La chirurgie de $\Gamma$ cesophage et du cardia 138

Jones, F. Avery, Carcinoma of the Gall-Bladder $\quad 357$ 
Kemp, F. H., vide G. M. Ardran

Koskínen, Penttí, vide Pekka Brummer

Laser, S, und L. Blòchlinger, Gastritis phlegmonosa (Bcschreibung eines antibiotisch behandelten und geheilten Falles) 94

Lyon, Ernst, Gallbladder Disease - Chronic Relapsing Pancreatitis - Diabetes

Mellitus 282

Mahlo, A., Die Bedeutung der Aktionsströme des Magens 227

Markoff, TV., Über die Klinik und Bedeutung der Perigastritis deformans ...

Nicole, R., Zur Frage des arteriomesenterialen Darmverschlusses 101

- Zur Behandlung der Osophagusatresien beim Neugeborenen 146

Norpoth, Leo, vide Joseph Cloesges

Oeler, Valerie, Sprue. Pathologisch-anatomische Untersuchungen bei 7 Beob-

achtungen von einheimischer und symptomatischer Sprue 257

Rechnitzer, P., Three Cases of Small Intestinal Neoplasm 343

Richards, W. C. D., vide J. Badenoch

Richards, W. C. D., vide G. M. Ardran

Richardson, J. E., Two Unusual Small Intestinal Neoplasms, both with

Anaemia $\quad 345$

Sarasin, Raymond, Les ulcères peptiques après gastrcctomie $\quad 148$

Schofield, B., vide C. C. Ungley Thompson, R. B., vide C. C. Ungley Truelove, S. C, vide G. M.

Ardran

Ungley, C. C. (in collaboration with E. V. Cox, B. Schofield and R. B. Thomp

son), Megaloblastic Anaemia Following Operations on the Intestine 338

Wagstaff, J. K., Demonstration - Post-Mortem Arteriography of the Normal

Stomach 378

Wang, H. W., Recherches sur les terminaisons nerveuses dans le foie

220

Watkínson, G., vide G. N. Chandler

Weiss, Ernst, Der heutige Stand der Vagotomiefrage beim Magen und Duodenalulcus 194

Wíssmer, Bernard, La cholinestérase sérique dans le diagnostic différentiel des ictères 111

Wilts, L. J., Haemopoiesis in Relation to Disorders of the Alimentary Tract . 322

$\mathrm{Ka}^{5} / 8$ uistik - Case Report $-<$ íasuistique,

Van Eeckhoutte, P., Cancer du versant gastrique de la gastro-entérostomie ... $41<$ iíesellscliaftsbei $\cdot$ iclite $-\mathrm{S}$ 'K'iety Transactions - Soeietes

Hie Congrès Européen des Sociétés Nationales de Gastro-Entérologie, Bologne

et Montecatini, 20-25 avril $1952 \quad 45$

Société beige de Gastro-entérologie 49

Schweizerische Gesellschaft für Gastroenterologie. Jahresversammlung in

Zurich vom 24. und 25. März 1952151

British Society of Gastroenterologists. Annual Meeting at London. Friday,

November 7th and Saturday, November 8th, 1952321

Editorial

Eosinophilie et parasitoses $\quad 44$

Les interventions sur Tartère hépatique dans le traitement des maladies du foie $\quad 156$

La carence protidique des gastrectomisés 
L'invagination sigmoïdo-rectale

Referate - Abstracts - Analyses

I. Allgemeines - Généralités

54

II. Mund - Bouche

57

II. Oesophagus 58

IV. Magen - Estomac 59,

157

V. Darm - Intestin

158

Physiologie, Pharmacologie

Sprue, Enteritis

158

Divertikel 163

Megacolon $\quad 165$

Colitis $\quad 166$

Appendicitis $\quad 171$

Infektionskrankheiten

Zirkulationsstörungen

Polyposis

177

11. Carcinoma 178

Rectum 180

Varia 182

VI. Leber-Foie $\quad 238, \quad 309$

Pathologische Physiologie

Diagnose 240

Die Leberbiopsie

Funktionsprüfungen

Hepatitis epidemica

243

Leberzirrhose

Krankheitsbild

244

Ascites 251

Infektionskrankheiten

Therapie

310

Carcinom

313

Hepatitis cystica

Diabetes und Leber

10. Varia

315

Buchbesprecliiiiiaen - Book Reviews - Mvres it $\notin n v \not a n x$ 62, 254, 317, 380 Varia 64, 256, 320

Alle Rechte vorbehalten

Printed in Switzerland

Buchdruckerei Karl Werner AG. in Basel 